\title{
BMJ Open Prognostic factors in metastatic breast cancer: a prospective single-centre cohort study in a Finnish University Hospital
}

\author{
Peeter Karihtala (D) , ${ }^{1,2}$ Anniina Jääskeläinen, ${ }^{1}$ Nelli Roininen, ${ }^{1}$ Arja Jukkola ${ }^{3}$
}

To cite: Karihtala $P$, Jääskeläinen $A$, Roininen $N$, et al. Prognostic factors in metastatic breast cancer: a prospective single-centre cohort study in a Finnish University Hospital. BMJ Open 2020;10:e038798. doi:10.1136/ bmjopen-2020-038798

- Prepublication history for this paper is available online. To view these files, please visit the journal online (http://dx.doi. org/10.1136/bmjopen-2020038798).

Received 24 March 2020 Revised 24 August 2020 Accepted 03 September 2020

\section{Check for updates}

(c) Author(s) (or their employer(s)) 2020. Re-use permitted under CC BY-NC. No commercial re-use. See rights and permissions. Published by BMJ.

${ }^{1}$ Department of Oncology and Radiotherapy, Medical Research Center Oulu, Oulu University Hospital and University of Oulu, Oulu, Finland

${ }^{2}$ Department of Oncology, Helsinki University Hospital Comprehensive Cancer Centre and University of Helsinki, Helsinki, Finland

${ }^{3}$ Department of Oncology, Tampere University Hospital, Cancer Center, Faculty of Medicine and Health Technology, University of Tampere, Tampere, Finland

Correspondence to Dr Peeter Karihtala; peeter.karihtala@helsinki.fi

\section{ABSTRACT}

Objectives Although novel early breast cancer prognostic factors are being continuously discovered, only rare factors predicting survival in metastatic breast cancer have been validated. The prognostic role of early breast cancer prognostic factors in metastatic disease also remains mostly unclear.

Design and setting Prospective cohort study in a Finnish University Hospital.

Participants and outcomes 594 women with early breast cancer were originally followed. Sixty-one of these patients developed distant metastases during the followup, and their primary breast cancer properties, such as tumour size, nodal status, oestrogen receptor (ER) and progesterone receptor expression, grade, proliferation rate, histopathological subtype and breast cancer subtype were analysed as potential prognostic factors for metastatic disease.

Results In multivariate analysis, the presence of lymph node metastases at the time of early breast cancer surgery $(\mathrm{HR}, 2.17 ; 95 \% \mathrm{Cl}, 1.09-4.31 ; \mathrm{p}=0.027)$ and $\mathrm{ER}$ status (negative vs positive, $\mathrm{HR}, 2.16 ; 95 \% \mathrm{Cl}, 1.14-4.10$; $\mathrm{p}=0.018$ ) were significant predictors of survival in metastatic disease.

Conclusions These results confirm ER status as a primary prognostic factor in metastatic breast cancer. Furthermore, it also suggests that the presence of initial lymph node metastases could serve as a prognostic factor in recurrent breast cancer.

\section{INTRODUCTION}

Breast cancer is by far the most common and deadliest cancer affecting women worldwide. ${ }^{1}$ In contrast to considerably prolonged early breast cancer prognosis during the last decade, which is mainly due to the optimisation of adjuvant therapies, the prognosis of patients with metastatic breast cancer has hardly been prolonged, and the current median of overall survival is approximately 36 months. $^{2-5}$

The presence of nodal involvement is the strongest predictor of outcomes for early breast cancer. ${ }^{6}$ The clinical behaviour of metastatic breast cancer still varies greatly,

\section{Strengths and limitations of this study}

- This study included contemporary, prospective breast cancer cohort in a University Hospital with a relatively long follow-up.

- The material did not include patients with de novo metastatic breast cancer.

- If the number of patients with metastatic breast cancer would have been larger, more detailed subgroup analyses, regarding, for example, biological subgroups possible, would have been possible.

and it is difficult to predict. The best validated prognostic factors in metastatic breast cancer include clinical factors, such as long relapse-free intervals, the absence of brain metastases or visceral metastases and the presence of oestrogen receptor (ER), which also serves as an essential predictive factor in metastatic settings. ${ }^{7-12}$ De novo metastatic breast cancers also have better prognosis than recurrent breast cancer. ${ }^{1013}$ The possibility of using other characteristics of primary breast cancer, such as primary tumour size and axillary lymph node status as prognostic factors in metastatic breast cancer is still being discussed; however, this approach has seldom been studied in modern prospective cohorts.

Using a large prospective breast cancer cohort treated with modern treatment modalities, we aimed to determine whether primary breast cancer prognostic factors, such as tumour size, nodal status, ER and progesterone receptor (PR) expression, differentiation, proliferation rate or breast cancer subtype could also predict outcomes in recurrent metastatic breast cancer.

\section{MATERIALS AND METHODS}

The original patient material was from a prospective dataset collected in Oulu University Hospital from 2003 to 2013. The dataset 


\begin{tabular}{|l|}
\hline Prospective cohort of invasive breast \\
cancer patients treated with breast \\
surgery \\
$\mathrm{n}=621$
\end{tabular}

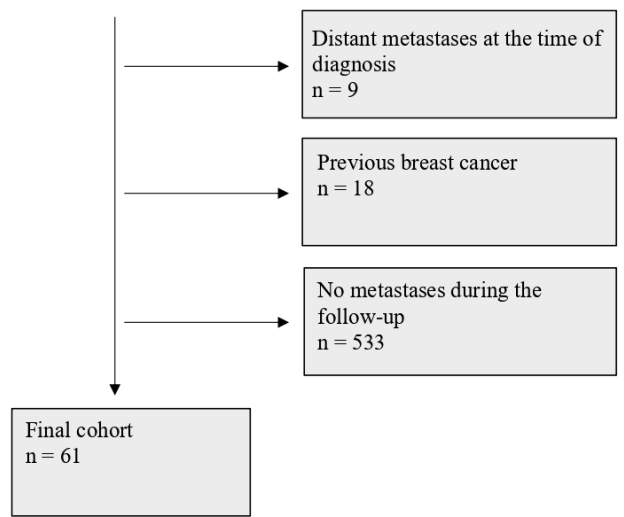

Figure 1 Flow chart of the study participants.

consisted of 594 patients with early invasive breast cancer diagnosed and treated in Oulu University Hospital, Finland. Surgery to the primary tumour was carried out according to the guidelines of the Finnish Breast Cancer Group. The dataset did not include information of the possible neoadjuvant chemotherapy, which was nevertheless very rarely administered during the study period. Patients with previous breast cancer or distant metastases at the time of diagnosis were excluded from the cohort (figure 1). During the follow-up, 61 women displayed distant metastases, and the outcomes of these patients were reported in this study.

Tumours were classed into five intrinsic subtypes according to the European Society for Medical Oncology (ESMO) clinical practice guidelines on breast cancer. ${ }^{14}$ Luminal A-like carcinomas expressed ER and PR, showed Ki-67 expression in $<15 \%$ of the cells, and did not overexpress human epidermal growth factor receptor 2 (HER2). Luminal B-like (HER2-negative) carcinomas were ER-positive and HER2-negative. In addition, they showed either Ki-67 expression in $\geq 15 \%$ of cells, or they were PR-negative. Luminal B-like (HER2-positive) tumours expressed ER and overexpressed HER2. Triple-negative breast carcinomas (TNBCs) were defined as tumours with no ER, PR and HER2 expression. HER2-positive (non-luminal) cases overexpressed HER2 without ER or PR positivity. The distribution between subtypes in the cohort is described in detail in table 1.

The histopathology was evaluated according to current WHO classification and stage was assessed using tumour, node, metastases (TNM) classification. The expressions of ER, PR and Ki-67 were studied using immunohistochemistry as previously described. ${ }^{15}$ HER2 expression was studied using immunohistochemistry and chromogenic in situ hybridisation (CISH) to confirm positive results. A positive result of six or more gene copies in CISH was considered HER2-positive. ${ }^{16}$
Table 1 Primary tumour characteristics

n (\%)

\begin{tabular}{|cc|}
\hline Tumour size & $20(32.8)$ \\
\hline T1 & $33(54.1)$ \\
T2 & $7(11.5)$ \\
\hline T3 & $1(1.6)$ \\
\hline T4 & \\
\hline Nodal status & $16(26.2)$ \\
\hline N0 1 & $22(36.1)$ \\
\hline N2 & $15(24.6)$ \\
\hline N3 & $8(13.1)$ \\
\hline Histopathology & \\
\hline Ductal & $47(77.0)$ \\
\hline Lobular & $11(18.0)$ \\
\hline Other & $3(4.9)$ \\
\hline
\end{tabular}

Histopathological grade

$\begin{array}{cc}\text { Grade } 1 & 0(0) \\ \text { Grade } 2 & 25(41.0) \\ \text { Grade } 3 & 34(55.7)\end{array}$

Unknown

$2(3.3)$

ER expression

$\begin{array}{lc}\text { Negative }(0 \%) & 14(23.0) \\ \text { Weak }(1 \%-9 \%) & 2(3.3) \\ \text { Moderate }(10 \%-59 \%) & 6(9.8)\end{array}$

High (>59\%) $39(63.9)$

PR expression

Negative (0\%) $22(36.1)$

Weak (1\%-9\%) $5(8.2)$

Moderate (10\%-59\%)

$5(8.2)$

High (>59\%)

29 (47.5)

HER2 status

$\begin{array}{lr}\text { HER2-negative } & 52(85.2) \\ \text { HER2-positive (CISH) } & 9(14.8)\end{array}$

Ki-67 expression

Negative (<5\%)

2 (3.3)

Weak (5\%-14\%) 15 (24.6)

Moderate (15\%-30\%)

20 (32.8)

High (>30\%) 24 (39.3)

Focality

$\begin{array}{ll}\text { Unifocal } & 50(82.1) \\ \text { Multifocal } & 11(18.0)\end{array}$

Subtype

Luminal A-like

$13(21.3)$

Luminal B-like (HER2-negative) 29 (47.5)

Luminal B-like (HER2-positive) 5 (8.2) HER2-positive, non-luminal 


\begin{tabular}{lc} 
Table 1 Continued & \\
\hline & $\mathbf{n}(\%)$ \\
\hline Triple-negative & $10(16.4)$ \\
Unknown & $1(1.6)$ \\
The first site of the distant metastasis \\
Bone only \\
Lung only & $17(27.9)$ \\
Liver only & $9(14.8)$ \\
Other & $5(8.2)$ \\
Multiple sites & $6(9.6)$ \\
\hline
\end{tabular}

$\mathrm{CISH}$, chromogenic in situ hybridisation; ER, oestrogen receptor; HER2, human epidermal growth factor receptor 2; PR, progesterone receptor.

\section{Statistical analyses}

Statistical analysis was performed using IBM SPSS Statistics software, V.25.0.0.0 for Mac (IBM Corporation). Survival was analysed with Kaplan-Meier curves and the log-rank tests. Correction for multiple comparisons was not made. Survival in metastatic disease was calculated from the date when metastasis was first observed in imaging to the time of death. Multivariate analysis was conducted using Cox multivariate regression analysis. $\mathrm{P}$ values $<0.05$ were considered significant.

\section{Patient and public involvement}

Patients or public were not involved in the design, conduct, reporting or dissemination of this study.

\section{RESULTS}

Sixty-one patients of the originally 594 women ultimately developed distant metastases during the follow-up. Of these, 50 patients died of breast cancer during the follow-up. The median disease-free interval was 39.0 months in the patients who had distant metastases. The median follow-up time starting from the early breast cancer diagnosis was 72.0 months in patients who later developed metastases.

The median follow-up of the patients during their metastatic breast cancer was 18.0 months (mean 30.2 months). The Kaplan-Meier estimate for median survival of the patients with metastatic breast cancer was 77.0 months in those with luminal A-like breast cancers, 29.0 months in those with luminal B-like (HER2-negative) disease and 11.0, 26.0 and 12.0 months in those with HER2-positive, non-luminal, luminal B-like (HER2-positive) and TNBC subtype, respectively.

Patients with metastatic local lymph nodes at the time of definitive surgery displayed poorer survival outcomes for metastatic disease $(p=0.031)$ (figure 2$)$. The KaplanMeier estimate for median survival in metastatic disease in lymph node-negative patients was 33.0 months, and in lymph node-positive patients, it was 19.0 months. Only N0 versus N1-3 classification was significant. No prognostic differences between the patients with $\mathrm{N} 1, \mathrm{~N} 2$ or N3 disease subtypes $(p=0.78)$ were detected.

Of the more traditional prognostic factors related to metastatic disease, ER positivity of the primary tumour ( $\mathrm{p}=0.011$ ), Ki-67 expression ranging from $0 \%$ to $14 \%$ (vs over $14 \%)$ in primary tumours $(\mathrm{p}=0.032)$ and grades I-II (vs grade III) primary tumours $(\mathrm{p}=0.012)$ were associated with better survival in metastatic disease in univariate analysis. Breast cancer subtype (determined from the initial surgical samples) also predicted survival with metastatic breast cancer ( $p=0.0078)$, Also, the patients with luminal A-like breast cancer had significantly prolonged survival, when compared with all other subtypes $(\mathrm{p}=0.017)$. Primary tumour size, PR or HER2 expression, the site of the first metastasis in bone versus elsewhere, disease-free interval ( $\leq 24$ months vs $>24$ months) or age at disease onset were not associated with metastatic disease survival.

When assessed separately by different biological subtypes, initial lymph node metastases predicted worse prognosis only in the patients with the luminal A subtype in univariate analysis $(p=0.019)$, but the small sample size of each subgroup limited the reliability of this analysis (data not shown).

In multivariate analysis, the presence of lymph node metastases at the time of initial diagnosis predicted poorer survival overall (HR, 2.17; 95\% CI, 1.09-4.31; $\mathrm{p}=0.027$ ) when tumour size (T1 vs T2-4) (HR, 1.33; 95\% CI, 0.712.47; $\mathrm{p}=0.37$ ) and ER status (negative vs positive) (HR, 2.16; 95\% CI, 1.14-4.10; $\mathrm{p}=0.018$ ) were included in the analysis. The proportional hazards assumption was met in the analysis. Breast cancer subtype, Ki-67 expression or grade did not remain significant prognostic factors after multivariate analysis.

\section{DISCUSSION}

As the main observation, we report in this prospectively collected and contemporary cohort from a Finnish University Hospital that the presence of local lymph node metastasis at the time of early breast cancer surgery predicted short survival in subsequent metastatic breast cancer. Our results also supported previous results of ER negativity in primary breast cancer as an adverse prognostic factor for disease recurrence.

The most established prognostic factors of better outcome in metastatic breast cancer include ER positivity, long disease-free interval (usually defined as at least 2 years), low number of metastatic sites and bone-only localisation of metastases. ${ }^{7-13}$ 17-19 HER2 appears to no longer represent a prognostic factor in the era of targeted treatments, and prognostic factors also slightly differ between HER2-positive and HER2-negative patients. ${ }^{20}{ }^{21}$ Emerging metastatic breast cancer prognostic factors include circulating tumour cells, gene expression panels, circulating tumour markers and miRNAs; however, they have not yet been sufficiently validated. ${ }^{22-25}$ Whereas ER status, a lengthy disease-free interval and metastatic load are established and obvious prognostic factors for metastatic 

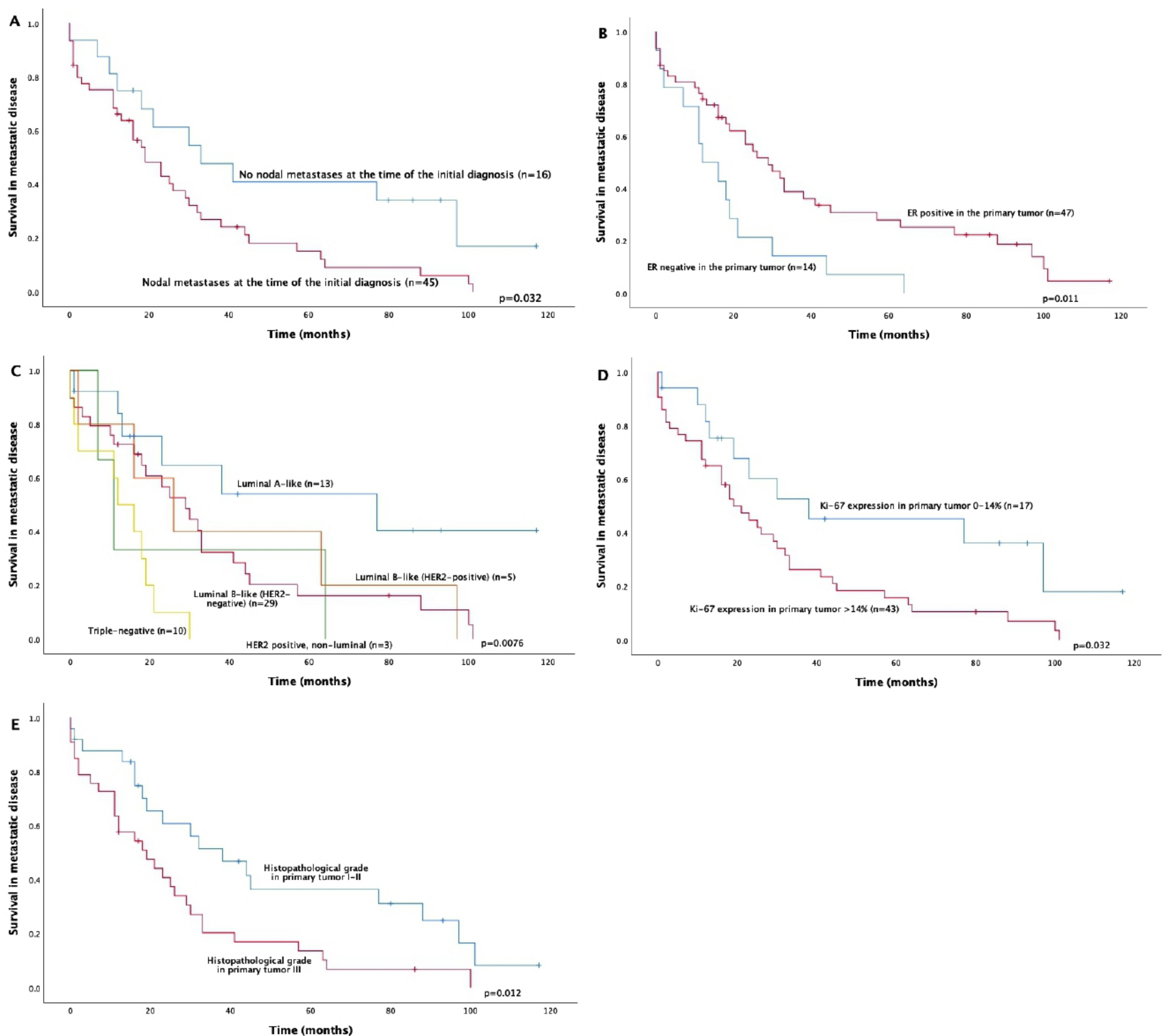

Figure 2 Associations between primary tumour properties and survival in metastatic breast cancer. In multivariate analysis, only ER expression and initial nodal status remained as significant factors. ER, oestrogen receptor; HER2, human epidermal growth factor receptor 2 .

breast cancer, the presence of lymph node metastases at the time of initial diagnosis has not been widely studied in metastatic breast cancer, although it is the strongest prognostic factor in early breast cancer. In the current study, we concentrated solely on primary breast cancer characteristics, and we did not assess other characteristics, such as disease-free interval, metastasis load or metastasis location as prognostic factors.

Some previous studies with mostly retrospective cohort settings and outdated treatment modalities have reported the initial nodal status as a prognostic factor in metastatic breast cancer, whereas others have not found such an association. ${ }^{792627}$ In the pioneer work of Clark et al, nodal involvement at time of initial diagnosis was associated with shorter survival. ${ }^{12}$ Another retrospective single-institute study also concluded that lymph node involvement at primary diagnosis predicted unfavourable outcomes in metastatic breast cancer, although the first patients were enrolled in the study cohort in the 1960s. ${ }^{28}$ In line with these studies, a Spanish retrospective registry study suggested that the initial lymph node status should be part of the prognostic index in recurrent metastatic breast cancer. ${ }^{29}$ In addition to considerable change in the oncological treatments of breast cancer during the last decades, also surgical techniques, especially axillary procedures have developed considerably. The current results from the prospective data with modern treatments thus support and confirm earlier results.

In our study, any metastasis found in local lymph nodes at the time of definitive surgery was associated with dismal 
metastatic cancer survival outcomes. The Kaplan-Meier estimate for median survival was prolonged from 19 to 33 months in patients without lymph node metastases at the time of initial diagnosis. Although lymph node metastases in general are associated with other factors of poor prognosis, our study suggested that this result was independent of tumour size and ER expression. Node positivity may reflect not only higher metastatic potential of breast cancer, but it can possibly decipher impaired immunological microenvironments. ${ }^{30}$ Interestingly, a recent paper by Ullah et al using evolutionary genomic analyses of primary tumours and metastatic lesions suggested that ipsilateral axillary lymph node status in primary breast cancer was very useful for predicting the tumourigenic capability of the primary tumour; however, it did not drive metastasis per se. ${ }^{31}$ Several other papers have suggested that metastatic lymph nodes did not eventually metastasise. ${ }^{32} 33$ However, it was also recently shown that the removal of metastatic axillary lymph nodes resulted in the disappearance of circulating tumour DNA, and discussion on these issues continues. ${ }^{34}$

It has to be emphasised that all our patients had recurrent breast cancer, and our material did not include samples from patients with de novo metastatic breast cancer. Whereas this makes the material more uniform, the results may not be suitable for generalising to de novo metastatic breast cancers, which have a different natural course from recurrent breast cancers. ${ }^{10} 13$ Nevertheless, the prognostic value of ER status has also been previously demonstrated in recurrent breast cancer, and the initial lymph node status obviously cannot be evaluated in de novo metastatic cancers. ${ }^{89} 18$ As an other limitation, we were unable to address the results separately in subgroups, for example, according to biological subtypes, due to relatively low number of patients with metastatic breast cancer. On the other hand, our study was based on a prospective cohort from a university clinic, and the patients were treated with up-to-date surgical and oncological treatment modalities.

Our results confirmed that ER negativity in primary tumour was associated with short survival for metastatic disease. This obviously is not only due to the more aggressive nature of the cancer but also because of the lack of ER-targeted treatments. Compelling evidence has demonstrated ER negativity in the primary tumour as an adverse prognostic factor in various previous studies. 89121318 ER status frequently changes in metastatic breast cancer, and the negative conversion of ER status is also a predictor of poor prognosis. ${ }^{35}$ Most previous studies have divided metastatic breast cancers only to three subgroups: ER/PR-positive, HER2-positive and TNBC. We used the widely recognised ESMO guidelines for subtyping our cases. Although the number of patients in each subgroup was rather limited, the patients with slowly proliferating, ER-responsive luminal A-like breast cancers still had significantly prolonged survival in metastatic breast cancer compared with other subtypes. TNBC has the worst outcome of all subtypes in metastatic breast cancer, a finding which was mirrored in our study. ${ }^{36}$

Predicting the course of metastatic breast cancer is of primary importance in clinical practice; however, its status as a highly heterogenous disease at both the intrapatient and interpatient levels makes metastatic breast cancer very unpredictable. ${ }^{31}$ Current metastatic breast cancer guidelines recommend starting treatment with chemotherapy or even with a combination chemotherapy instead of hormonal treatments in patients with visceral crisis or rapidly progressing ER-positive, HER2-negative breast cancer. ${ }^{2}{ }^{38}$ If novel adverse prognostic factors of metastatic breast cancer, such as initial nodal status, could be confirmed, these patients should receive more aggressive first-line metastatic breast cancer therapy.

In conclusion, our results strengthen the role of primary tumour ER negativity as an adverse prognostic factor in patients with recurrent breast cancer; however, they also suggest that initial lymph node status may be a prognostic factor for metastatic disease course. Future studies should also evaluate the prognostic power of isolated tumour cells, micrometastases and the absolute number of metastatic lymph nodes, which were not addressed in our material. More research is also clearly needed to clarify whether axillary lymph node metastases are able to seed metastatic cells or whether they are purely an indicator of aggressive disease.

Contributors All authors contributed to the study design and conception. AJu initiated the collection of the prospective dataset. PK, AJä and NR were responsible for assessing statistical analyses. PK was a major contributor in writing the manuscript. All authors provided comments on drafts of the manuscript. All authors read and approved the final manuscript.

Funding The authors have not declared a specific grant for this research from any funding agency in the public, commercial or not-for-profit sectors.

Competing interests None declared.

Patient consent for publication Not required.

Ethics approval The patients provided their written informed consent to participate the study. The study was approved by the Local Ethics Committee of the Ostrobothnia Hospital District (114/2011) and the National Supervisory Authority for Welfare and Health (D9580/05.01.00.06/2010). All studies were conducted in accordance with the principles of the Declaration of Helsinki and the guidelines for good clinical practice.

Provenance and peer review Not commissioned; externally peer reviewed. Data availability statement Patient data are available upon reasonable request. Open access This is an open access article distributed in accordance with the Creative Commons Attribution Non Commercial (CC BY-NC 4.0) license, which permits others to distribute, remix, adapt, build upon this work non-commercially, and license their derivative works on different terms, provided the original work is properly cited, appropriate credit is given, any changes made indicated, and the use is non-commercial. See: http://creativecommons.org/licenses/by-nc/4.0/.

\section{ORCID iD}

Peeter Karihtala http://orcid.org/0000-0003-3490-3702

\section{REFERENCES}

1 Bray F, Ferlay J, Soerjomataram I, et al. Global cancer statistics 2018: GLOBOCAN estimates of incidence and mortality worldwide for 36 cancers in 185 countries. CA Cancer J Clin 2018;68:394-424.

2 Cardoso F, Senkus E, Costa A, et al. 4th ESO-ESMO International Consensus Guidelines for Advanced Breast Cancer (ABC 4)†. Ann Oncol 2018;29:1634-57. 
3 Cardoso F, Spence D, Mertz S, et al. Global analysis of advanced/ metastatic breast cancer: decade report (2005-2015). Breast 2018;39:131-8.

4 Allemani C, Weir HK, Carreira H, et al. Global surveillance of cancer survival 1995-2009: analysis of individual data for $25,676,887$ patients from 279 population-based registries in 67 countries (CONCORD-2). Lancet 2015;385:977-1010.

5 Weigelt B, Peterse JL, van 't Veer LJ. Breast cancer metastasis: markers and models. Nat Rev Cancer 2005;5:591-602.

6 Peto R, Davies C, Pritchard K, et al. Comparisons between different polychemotherapy regimens for early breast cancer: meta-analyses of long-term outcome among 100,000 women in 123 randomised trials. Lancet 2012;379:432-44.

7 Chang J, Clark GM, Allred DC, et al. Survival of patients with metastatic breast carcinoma: importance of prognostic markers of the primary tumor. Cancer 2003;97:545-53.

8 Regierer AC, Wolters R, Ufen M-P, et al. An internally and externally validated prognostic score for metastatic breast cancer: analysis of 2269 patients. Ann Oncol 2014;25:633-8.

9 Largillier R, Ferrero J-M, Doyen J, et al. Prognostic factors in 1,038 women with metastatic breast cancer. Ann Oncol 2008;19:2012-9.

10 Shen T, Gao C, Zhang K, et al. Prognostic outcomes in advanced breast cancer: the metastasis-free interval is important. Hum Pathol 2017;70:70-6.

11 Stuart-Harris R, Shadbolt B, Palmqvist C, et al. The prognostic significance of single hormone receptor positive metastatic breast cancer: an analysis of three randomised phase III trials of aromatase inhibitors. Breast 2009;18:351-5.

12 Clark GM, Sledge GW, Osborne CK, et al. Survival from first recurrence: relative importance of prognostic factors in 1,015 breast cancer patients. J Clin Oncol 1987;5:55-61.

13 Lobbezoo DJA, van Kampen RJW, Voogd AC, et al. Prognosis of metastatic breast cancer: are there differences between patients with de novo and recurrent metastatic breast cancer? $\mathrm{Br} \mathrm{J}$ Cancer 2015;112:1445-51.

14 Senkus E, Kyriakides S, Ohno S, et al. Primary breast cancer: ESMO clinical practice guidelines for diagnosis, treatment and follow-up. Ann Oncol 2015;26 Suppl 5:v8-30.

15 Karihtala P, Mäntyniemi A, Kang SW, et al. Peroxiredoxins in breast carcinoma. Clin Cancer Res 2003;9:3418-24.

16 Isola J, Tanner M, Forsyth A, et al. Interlaboratory comparison of HER-2 oncogene amplification as detected by chromogenic and fluorescence in situ hybridization. Clin Cancer Res 2004;10:4793-8

17 Yamamoto N, Watanabe T, Katsumata N, et al. Construction and validation of a practical prognostic index for patients with metastatic breast cancer. J Clin Oncol 1998;16:2401-8.

$18 \mathrm{Kim} \mathrm{H}$, Choi DH, Park W, et al. Prognostic factors for survivals from first relapse in breast cancer patients: analysis of deceased patients. Radiat Oncol J 2013;31:222-7.

19 Khanfir A, Lahiani F, Bouzguenda R, et al. Prognostic factors and survival in metastatic breast cancer: a single institution experience. Rep Pract Oncol Radiother 2013;18:127-32.

20 Hopkins AM, Rowland A, McKinnon RA, et al. Predictors of longterm disease control and survival for HER2-positive advanced breast cancer patients treated with pertuzumab, trastuzumab, and docetaxel. Front Oncol 2019;9:789.
21 Dawood S, Broglio K, Buzdar AU, et al. Prognosis of women with metastatic breast cancer by HER2 status and trastuzumab treatment: an institutional-based review. J Clin Oncol 2010;28:92-8.

22 King TA, Lyman JP, Gonen M, et al. Prognostic impact of 21-gene recurrence score in patients with stage IV breast cancer: TBCRC 013. J Clin Oncol 2016;34:2359-65.

23 Prat A, Cheang MCU, Galván P, et al. Prognostic value of intrinsic subtypes in hormone receptor-positive metastatic breast cancer treated with letrozole with or without lapatinib. JAMA Oncol 2016;2:1287-94.

24 Papadaki C, Stoupis G, Tsalikis L, et al. Circulating miRNAs as a marker of metastatic disease and prognostic factor in metastatic breast cancer. Oncotarget 2019;10:966-81.

25 Van Poznak C, Somerfield MR, Bast RC, et al. Use of biomarkers to guide decisions on systemic therapy for women with metastatic breast cancer: American Society of clinical oncology clinical practice guideline. J Clin Oncol 2015;33:2695-704.

26 Insa A, Lluch A, Prosper F, et al. Prognostic factors predicting survival from first recurrence in patients with metastatic breast cancer: analysis of 439 patients. Breast Cancer Res Treat 1999;56:67-78.

27 Tsuji W, Teramukai S, Ueno M, et al. Prognostic factors for survival after first recurrence in breast cancer: a retrospective analysis of 252 recurrent cases at a single institution. Breast Cancer 2014;21:86-95.

28 Rack B, Janni W, Gerber B, et al. Patients with recurrent breast cancer: does the primary axillary lymph node status predict more aggressive tumor progression? Breast Cancer Res Treat 2003;82:83-92.

29 Puente J, López-Tarruella S, Ruiz A, et al. Practical prognostic index for patients with metastatic recurrent breast cancer: retrospective analysis of 2,322 patients from the GEICAM Spanish El Alamo register. Breast Cancer Res Treat 2010;122:591-600.

30 Gibert-Ramos A, López C, Bosch R, et al. Immune response profile of primary tumour, sentinel and non-sentinel axillary lymph nodes related to metastasis in breast cancer: an immunohistochemical point of view. Histochem Cell Biol 2019;152:177-93.

31 Ullah I, Karthik G-M, Alkodsi A, et al. Evolutionary history of metastatic breast cancer reveals minimal seeding from axillary lymph nodes. J Clin Invest 2018;128:1355-70.

32 Klein CA. Selection and adaptation during metastatic cancer progression. Nature 2013;501:365-72.

33 Engel J, Emeny RT, Hölzel D. Positive lymph nodes do not metastasize. Cancer Metastasis Rev 2012;31:235-46.

34 Barry P, Vatsiou A, Spiteri I, et al. The spatiotemporal evolution of lymph node spread in early breast cancer. Clin Cancer Res 2018;24:4763-70.

35 Woo JW, Chung YR, Ahn S, et al. Changes in biomarker status in metastatic breast cancer and their prognostic value. J Breast Cancer 2019;22:439-52.

36 Fietz T, Tesch H, Rauh J, et al. Palliative systemic therapy and overall survival of 1,395 patients with advanced breast cancer results from the prospective German TMK cohort study. The Breast 2017:34:122-30.

37 Januškevičienė I, Petrikaitè V. Heterogeneity of breast cancer: the importance of interaction between different tumor cell populations. Life Sci 2019;239:117009.

38 NCCN. Breast cancer, 2019. Available: https://www.nccn.org/ professionals/physician_gls/pdf/breast.pdf [Accessed 12 Jan 2019]. 